\title{
Tunable optical cavity for an x-ray free-electron-laser oscillator
}

\author{
Kwang-Je Kim and Yuri V. Shvyd'ko \\ Advanced Photon Source, Argonne National Laboratory, Argonne, Illinois 60439, USA
}

(Received 19 December 2008; published 5 March 2009)

\begin{abstract}
An x-ray free-electron laser oscillator proposed recently for hard $\mathrm{x}$ rays [K. Kim, Y. Shvyd'ko, and S. Reiche, Phys. Rev. Lett. 100, 244802 (2008)] can be made tunable by using an x-ray cavity composed of four crystals, instead of two. The tunability of x-ray energy will significantly enhance the usefulness of an X-ray free-electron laser oscillator. We present a detailed analysis of the four-crystal optical cavity and choice of crystals for several applications: inelastic x-ray scattering, nuclear resonant scattering, bulksensitive hard x-ray photoemission spectroscopy, other high-energy-resolution ( $\lesssim 1 \mathrm{meV}$ ) spectroscopic probes, and for imaging with hard $\mathrm{x}$ rays at near-atomic resolution $(\simeq 1 \mathrm{~nm})$.
\end{abstract}

DOI: $10.1103 /$ PhysRevSTAB.12.030703

PACS numbers: 41.60.Cr, 41.50.+h, 42.55.Vc, 61.05.C-

\section{INTRODUCTION}

$\mathrm{X}$ rays produced by electron beams propagating through undulators could be trapped by Bragg reflecting crystal mirrors giving rise to a new device called an x-ray freeelectron laser oscillator (XFELO), as was first discussed in [1]. Recently it was shown that the XFELO is feasible based on a low-loss x-ray crystal cavity and ultralowemittance electron beams [2]. The XFELO makes use of Bragg diffracting crystals as high-reflectivity mirrors together with ultralow-emittance electron beams available from a multi-GeV energy recovery linacs [3] or produced by a cw linac employing an ultralow-emittance injector [4]. Because of a narrow bandwidth $\simeq 2 \mathrm{meV}$, the peak spectral brightness of an XFELO is predicted to be comparable to that of the self-amplified-spontaneous emission (SASE) from a high-gain x-ray free-electron laser [5-7], while the average brightness is higher by several orders of magnitude.

A basic limitation of the two-crystal $\mathrm{x}$-ray cavity schemes discussed in Ref. [2] is that X-ray photon energy $E$ is not tunable. However, a cavity employing four crystals can be made tunable, as was first proposed by Cotterill 40 years ago $[8,9]$ and independently by one of us more recently [10]. In this paper we discuss in detail the fourcrystal tunable $\mathrm{x}$-ray cavity in the context of an XFELO and the choice of crystals for several important applications.

\section{TUNABLE CAVITIES}

Among the three schemes for cavity configuration considered in Ref. [2], the one consisting of two near-normalincidence crystals and a grazing-incidence mirror, shown schematically in Fig. 1, appears to be a candidate for a tunable cavity. Here, crystals are oriented so that the incident and exit x rays at each crystal form the same angle $\Theta$ with respect to the diffraction vector $\boldsymbol{H}$, normal to the reflecting atomic planes. The angles of incidence and reflection are equal. A grazing-incidence mirror is neces- sary to close the loop of the x-ray path and to focus onto the electron bunch in the undulator. The mirror is oriented so that the incident and exit $\mathrm{x}$ rays are symmetric to the normal of the mirror surface. The grazing angle of incidence on the mirror is then $2 \Theta$. The photon energy $E$ is determined by Bragg's law:

$$
E=E_{H} / \cos \Theta .
$$

Here, $E_{H}$ is the Bragg energy, the energy of photons reflected at normal incidence from the atomic reflecting planes with the diffraction vector $\boldsymbol{H}$. The angle of incidence is related to the Bragg angle via $\theta_{\mathrm{B}}=\pi / 2-\Theta$. The photon energy $E$ can be changed by changing $\Theta$, which is accomplished by changing the distance $G$ and reorienting the crystals to maintain Bragg reflection. However, the tuning range in this configuration is restricted by the requirement that the grazing angle of incidence $2 \Theta$ on the mirror be smaller than the critical angle $\theta_{\text {cr }}$ of total reflection. For photon energy $E \simeq 10 \mathrm{keV}$, typically $\theta_{\text {cr }} \simeq$ $1 \mathrm{mrad}$. This requirement turns out to be too strict to allow practically interesting tuning ranges. For example, the grazing angle of incidence for a perfect palladium mirror has to be less than $3 \mathrm{mrad}$ for mirror reflectivity to be higher than $90 \%$. Assuming angular variation $\delta \Theta \simeq$ $0.5 \mathrm{mrad}$, this would result in an energy variation of $\delta E \simeq$ $E_{H} \Theta \delta \Theta \simeq 7 \times 10^{-7} E_{H}$. For $E_{H}=10 \mathrm{keV}$, the energy

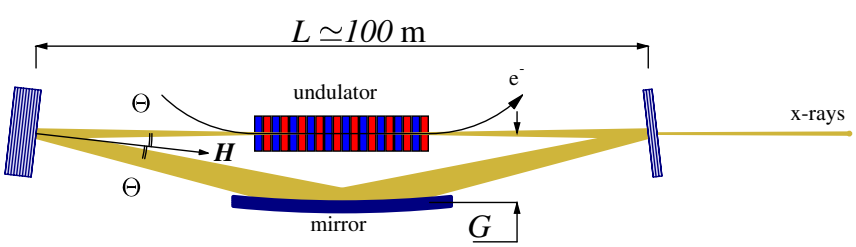

FIG. 1. (Color) Scheme of an X-ray cavity with two crystals at near-normal incidence. The grazing-incidence mirror focuses $\mathrm{x}$ rays onto the electron bunch $e^{-}$in the undulator and closes the loop of the x-ray path. This cavity configuration considered in [2] has a very limited tuning range, as explained in the text. 


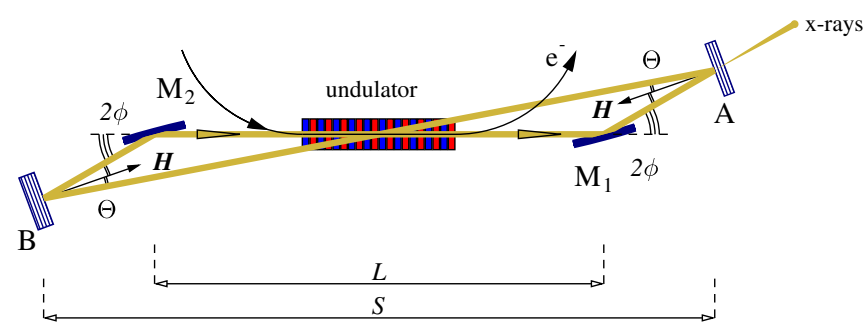

FIG. 2. (Color) Similar to the X-ray cavity in Fig. 1, but, with two mirrors: $M_{1}$ collimates the x-ray beam, while $M_{2}$ focuses $x$ rays onto the electron bunch $e^{-}$in the undulator. This cavity configuration has also a very restricted range of tunability because of the small grazing angle of incidence $\phi \simeq 1 \mathrm{mrad}$.

range of variation is then $\delta E \simeq 7 \mathrm{meV}$. The range would be even smaller for a silica mirror.

Figure 2 shows a similar configuration with two mirrors. This configuration could be more favorable for controlling the mode profile and optimizing the coupling of the $\mathrm{x}$ ray and the electron beams (see discussion that follows). A configuration with crystals A and B placed on the same side of the undulator could be also considered as an option. The tuning range is still limited in these cases, as well.

An x-ray cavity tunable in a broad energy range can be constructed by employing a four-crystal configuration as shown in Fig. 3, in which crystals A, B, C, and D are placed at the four corners of an isosceles trapezoid and the $\mathrm{x}$ rays propagate along the zigzag path [8-10]. The line AD passes through an undulator where the XFEL interaction takes place. The length $L$ of the path AD is long, about $100 \mathrm{~m}$ for examples considered in Ref. [2]. It would be convenient to fix the positions of $\mathrm{A}$ and $\mathrm{D}$ so that the length $L$ is constant. It would be also practical to choose the length $S$ of the path BC to be short, a few meters or less, so that crystals B and C could be placed on a single optical table. The crystals should be oriented so that the Bragg

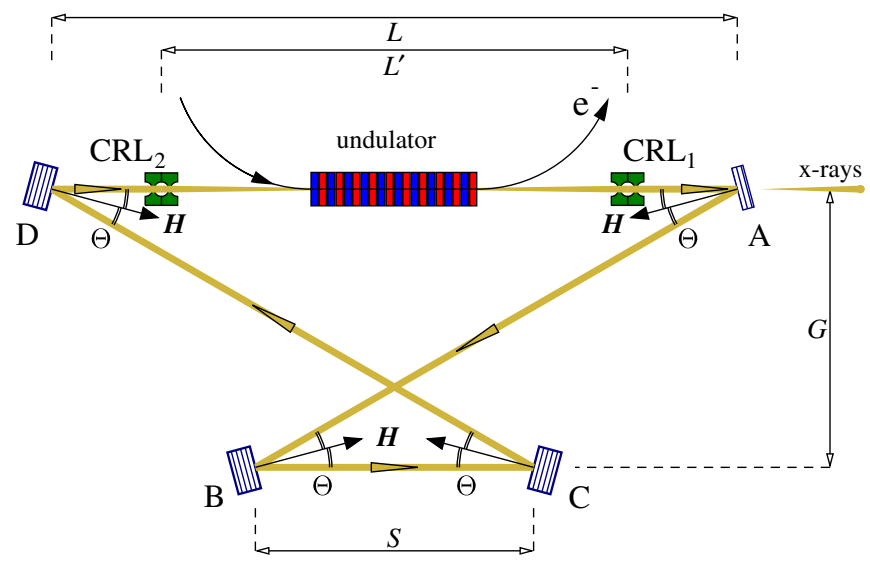

FIG. 3. (Color) A scheme of a four-crystal (A, B, C, and D) $\mathrm{X}$-ray optical cavity allowing a broad range of energy tuning assuming that the focusing elements are the compound refractive lenses (CRLs) [12]. See text for other details. condition is always satisfied for each crystal. The angle of incidence $\Theta$ is then the same at all crystals and related to the dimensions of the trapezoid by

$$
\tan 2 \Theta=\frac{2 G}{L+S},
$$

where $G$ is the distance between the two parallel paths $\mathrm{AD}$ and $\mathrm{BC}$. The four-crystal geometry requires that $\Theta<\pi / 4$. An equivalent crystal configuration is used in the so-called $(+--+)$ four-crystal $\mathrm{x}$-ray monochromator, and therefore, its theory could be applied to describe the single-pass spectral properties of the cavity (see, e.g., [11] for references and details).

The round-trip path length of $\mathrm{x}$ rays is given by

$$
\ell=(L+S) \frac{1+\cos 2 \Theta}{\cos 2 \Theta} .
$$

It is mandatory that $\ell$ does not change while tuning the cavity, since the round-trip time should be constant to a high accuracy and equal to the time interval between two adjacent electron bunches.

The $\mathrm{x}$-ray photon energy is tuned by changing $\Theta$, which in turn requires changing $G$ and $S$. The critical point here is that the lines $\mathrm{AD}$ and $\mathrm{BC}$ are parallel so that the angle $\Theta$ is the same at all four crystal locations. Combining Eqs. (2) and (3) we obtain

$$
\begin{gathered}
G=\frac{\ell}{2} \tan \Theta, \\
L+S=\frac{\ell}{2}\left(1-\tan ^{2} \Theta\right) .
\end{gathered}
$$

Equations (4) and (5), specify how $G$ and $S$ should vary as the angle $\Theta$ changes while tuning the cavity, with $\ell$ and $L$ assumed to be fixed parameters. To increase the photon energy $E$, the incident angle $\Theta$ needs to be increased. The distance $G$ will then have to be increased according to Eq. (4). Similarly the distance $S$ will have to be decreased according to Eq. (5). Let $E_{0}, \Theta_{0}$, and $G_{0}$ be the values of $E$, $\Theta$, and $G$, respectively, in the limit $S=0$. From Eq. (2) it follows that $\tan 2 \Theta_{0}=2 G_{0} / L$, Note that for a chosen value of $G_{0}, \Theta_{0}$ is the maximum incidence angle corresponding to the largest photon energy $E_{0}=E_{H} / \cos \Theta_{0}$ in the tuning range.

The angular variations $\delta \Theta$ will be small in most cases. The photon energy variation can then be written approximately as $\delta E / E=\tan \Theta_{0} \delta \Theta$ in view of Eq. (1). Using this expression together with Eqs. (4) and (5), the following equations can be derived:

$$
\delta G \simeq \frac{\ell}{2} \delta \Theta \simeq \frac{\ell}{2 \Theta_{0}} \frac{\delta E}{E}, \quad \delta S \simeq-\ell \Theta_{0} \delta \Theta \simeq-\ell \frac{\delta E}{E} .
$$

In deriving Eq. (6) we have assumed that $\Theta_{0} \lesssim 0.1$, as in the examples considered below. This equation gives an 
estimate of the change in $G$ and $S$ for a required energy variation $\delta E$ at small incidence angles. For example, a relative energy variation of $\delta E / E \simeq 10^{-4}$, in a cavity with $\ell \simeq 200 \mathrm{~m}$, would require variations in $G$ and $S$ to be $\delta G \simeq 10 / \Theta_{0} \mathrm{~mm}$ and $\delta S \simeq 20 \mathrm{~mm}$, respectively.

Focusing elements are necessary in the XFELO optical cavity to control the mode profile and to optimize the coupling of the $x$ ray and the electron beam. In Fig. 3 the focusing elements are assumed to be the compound refractive lenses (CRLs) [12]. Paraboloidal CRLs [13] made of Be would be most appropriate. However, polycrystalline Be CRLs currently employed at synchrotron radiation facilities are not suitable for an XFELO, because of the significant loss due to small angle scattering from the boundaries of the Be microcrystals. It should be possible in the future to manufacture low-loss CRLs applicable for XFELOs, from beryllium or diamond single crystals.

Another option is to use grazing-incidence ellipsoidal mirrors as focusing elements, choosing a small grazing angle of incidence $\phi \simeq 1 \mathrm{mrad}$ for high reflectivity, as shown in Fig. 4. In this case, we have to modify the definition of $L$ as the distance between the crystals A and D projected on the direction parallel to $\mathrm{AM}_{1}$; and $G$ as the distance between two parallel paths $\mathrm{AM}_{1}$ and $\mathrm{CB}$. Hence, the x-ray paths $\mathrm{DM}_{2}$ and $\mathrm{AM}_{1}$ are parallel to each other and to the path BC. However, the path $\mathrm{AM}_{1}$ is displaced from $\mathrm{DM}_{2}$ by a distance $\Delta G$. We assume that $\mathrm{DM}_{2}$ and $\mathrm{AM}_{1}$ have the same length, and the undulator is placed parallel to and in the middle of the line $\mathrm{M}_{1} \mathrm{M}_{2}$. The center of the undulator is displaced by a distance $F$ from both the midpoint between crystals $\mathrm{B}$ and $\mathrm{C}$, and the intersection of lines $\mathrm{CD}$ and $\mathrm{AB}$. This is necessary to make the incidence angle $\Theta$ the same for all crystals. The round-trip path length of $\mathrm{x}$ rays (which has to be kept fixed) is given in this case by

$$
\tilde{\ell}=\ell+\Delta \ell
$$

where $\ell$ is given by Eq. (3) and

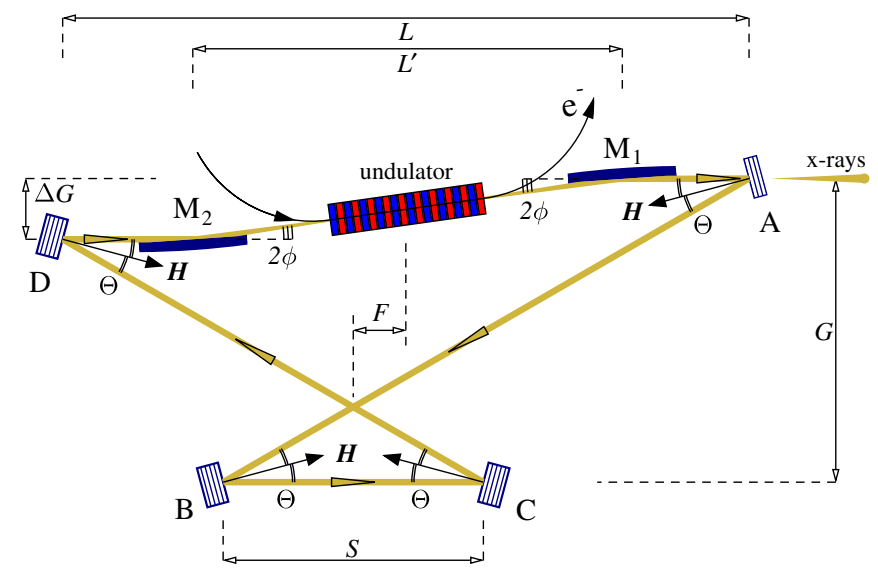

FIG. 4. (Color) Same as Fig. 3, with the ellipsoidal mirrors $\mathbf{M}_{1}$ and $\mathrm{M}_{2}$ used as collimating and focusing elements.

$$
\Delta \ell=L^{\prime}\left(\frac{1}{\cos 2 \phi}-1\right)
$$

Here $L^{\prime}$ is the distance between two mirrors projected on the line parallel to $\mathrm{AM}_{1}$. For small $\phi, \Delta \ell \approx 2 L^{\prime} \phi^{2}$ is very small: $\Delta \ell \approx 0.2 \mathrm{~mm}$ (assuming $L^{\prime} \approx 10^{5} \mathrm{~mm}$ ).

In addition to keeping the distance $L$ fixed, it would be convenient in the present case to hold constant the location and orientation of the mirrors $\mathrm{M}_{1}$ and $\mathrm{M}_{2}$, thus holding $\phi$ and $L^{\prime}$ fixed. Then the quantities $\Delta \ell$ and $\ell$ would be also kept constant. The cavity is tuned by changing $G, F$, and $S$ according to the following equations:

$$
\begin{gathered}
G=\frac{\ell}{2} \tan \Theta+\frac{\Delta G}{2}, \quad \Delta G=2 L^{\prime} \phi, \\
F=\Delta G /(2 \tan 2 \Theta), \quad L+S=\frac{\ell}{2}\left(1-\tan ^{2} \Theta\right) .
\end{gathered}
$$

\section{CONTROLLING ANGULAR DIVERGENCE OF $X$ RAYS AND ANGULAR ACCEPTANCE OF CRYSTALS}

We have to check whether the angular range available for energy tuning in the four-crystal cavity may be limited by the fact that increasing $\Theta$ results in an unfavorable decrease of the angular acceptance of the Bragg reflection. The angular acceptance is given by $\Delta \Theta^{(\mathrm{s})}=\epsilon_{H}^{(\mathrm{s})} / \tan \Theta$, see, e.g., [11]. Here $\epsilon_{H}^{(\mathrm{s})}$ is a relative energy width of the Bragg reflection with the diffraction vector $\boldsymbol{H}$, a quantity that is to a good accuracy invariant for a given Bragg reflection. In diamond crystals, which will be discussed in more detail below, the relative energy width varies from $\epsilon_{H}^{(\mathrm{s})} \simeq 10^{-5}$ for reflections with $E_{H} \simeq 5 \mathrm{keV}$, to $\epsilon_{H}^{(\mathrm{s})} \simeq 2 \times$ $10^{-7}$ for reflections with $E_{H} \simeq 25 \mathrm{keV}$, see Fig. 5. The

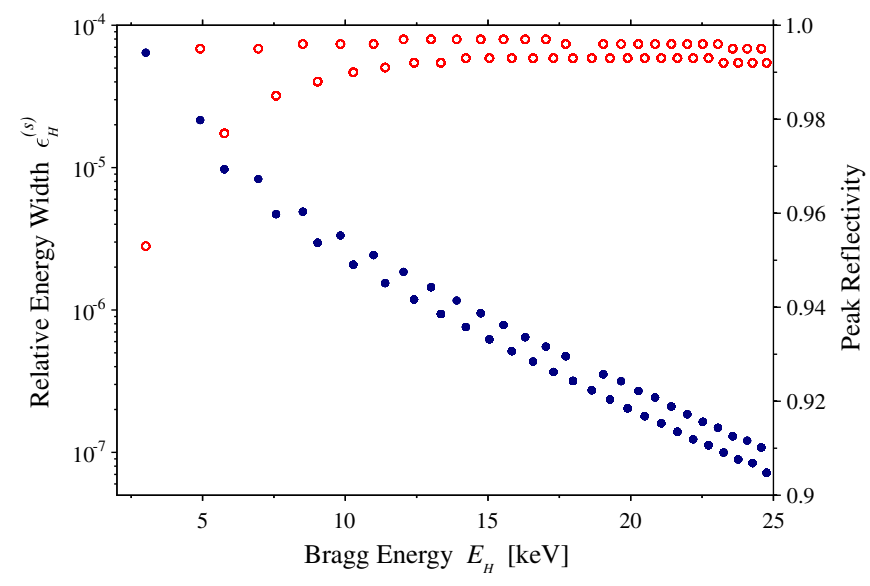

FIG. 5. (Color) Closed circles: relative energy width $\epsilon_{H}^{(\mathrm{s})}$ of all allowed Bragg reflections in diamond (C) crystals, in the symmetric scattering geometry, for Bragg energies $E_{H}$ up to $25 \mathrm{keV}$. Open circles: the same for the peak reflectivity. Calculations are performed with dynamical theory of x-ray diffraction in thick crystals as described in [11]. Debye-Waller factors are calculated using $2200 \mathrm{~K}$ Debye temperature. 
angular acceptance of the Bragg reflection $\Delta \Theta^{(\mathrm{s})}$ has to be compared with the angular divergence $\Delta \Theta_{\mathrm{X}}$ of $\mathrm{x}$ rays impinging on the crystal. To assure high reflectivity, one must require

$$
\Delta \Theta^{(\mathrm{s})} \gg \Delta \Theta_{\mathrm{X}} .
$$

This inequality determines the range of admissible angular variation of the crystals with the relative energy width $\epsilon_{H}^{(\mathrm{s})}$. The maximum angle is given by

$$
\tan \Theta_{\max } \ll \epsilon_{H}^{(\mathrm{s})} / \Delta \Theta_{\mathrm{X}} .
$$

The range of energy variation, taking into account Bragg's law (1) and (11), is

$$
E_{H} \leq E \leq E_{H} / \cos \Theta_{\max } .
$$

The angular divergence $\Delta \Theta_{\mathrm{X}}$ can be made small by properly designing the optical cavity layout, that is, by choosing suitable values for the focal lengths and positions of the two focusing elements in Figs. 3 and 4 . The x-ray path in the optical cavity is divided into two different regions: the interaction region containing the undulator and limited by two focusing elements and the free region containing the rest. The crystals are located in the free region. The angular divergence $\Delta \Theta_{\mathrm{XFEL}}$ of the x rays in the interaction region is chosen to maximize the XFEL gain. For $10 \mathrm{keV}$ radiation it is $\Delta \Theta_{\mathrm{XFEL}} \simeq 2.5 \mu \mathrm{rad}$ (FWHM) [2]. The angular divergence in the free region $\Delta \Theta_{X}$ is determined by the focal lengths and positions of the focusing elements. A reasonable cavity layout was obtained in [14] in which $\Delta \Theta_{\mathrm{X}}$ is 5 times less than $\Delta \Theta_{\mathrm{XFEL}}: \Delta \Theta_{\mathrm{X}}=$ $0.5 \mu \mathrm{rad}$. This number is also close to the presently achievable figure error in mirror fabrication, and thus the angular collimation achievable with grazing-incidence mirrors.

Although $\Delta \Theta_{\mathrm{X}}$ is small, Eq. (11) still imposes a limitation on the tunability range, especially significant for Bragg reflections with Bragg energy $E_{H}>15 \mathrm{keV}$, for which $\epsilon_{H}^{(\mathrm{s})} / \Delta \Theta_{\mathrm{X}}<1$. Indeed in this case $\Theta_{\max } \lesssim 0.1$, and therefore the tunability range according to Eq. (12) is about $10^{-2} E_{H}$. The current analysis shows that also in the case of the four-crystal cavity scheme the backscattering geometry is more favorable because of the constraints imposed by the small angular widths of Bragg reflections in off-backscattering geometry.

The angular acceptance can further be increased by using Bragg diffraction from cavity crystals in asymmetric scattering geometry. So far we have considered the symmetric scattering geometry, in which the reflecting atomic planes are parallel to the crystal surface. If there is a nonzero asymmetry angle $\eta$ between the crystal surface normal $\boldsymbol{n}$ and the diffraction vector $\boldsymbol{H}$, as shown in Fig. 6, the so-called asymmetric scattering geometry takes place, offering a possibility to vary the Bragg diffraction widths. The relative spectral width of the Bragg reflection becomes

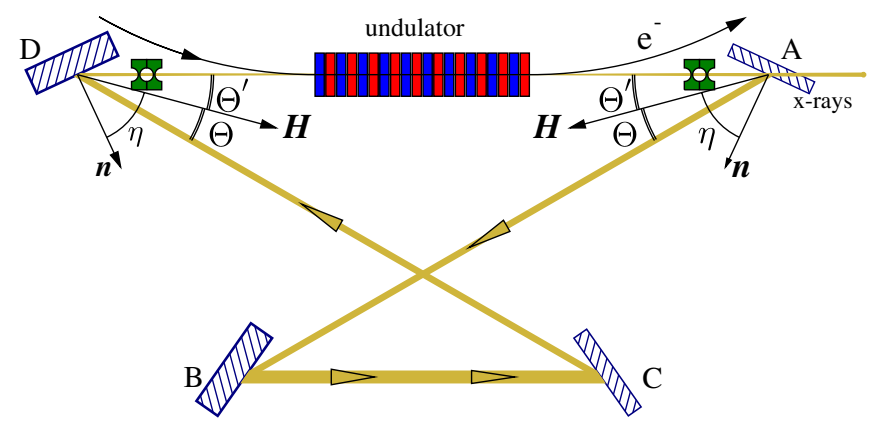

FIG. 6. (Color) Scheme of a four-crystal x-ray optical cavity with asymmetrically cut crystals (the crystal surface normal $\boldsymbol{n}$ makes a nonzero angle $\eta$ with the diffraction vector $\boldsymbol{H}$ ) allowing tailoring angular acceptance and spectral width of Bragg reflections. Note that the angles of incidence $\Theta$ and reflection $\Theta^{\prime}$ are no longer equal. The scattering geometry for $\mathrm{B}$ is the same as for $\mathrm{A}$, while the scattering geometry for $\mathrm{C}$ is the same as for $\mathrm{D}$.

$\epsilon_{H}=\epsilon_{H}^{(\mathrm{s})} / \sqrt{|b|}$, the angular acceptance becomes $\Delta \Theta=$ $\Delta \Theta^{(\mathrm{s})} / \sqrt{|b|}$, while the angular divergence of the reflected x rays becomes $\Delta \Theta^{\prime}=\Delta \Theta^{(\mathrm{s})} \sqrt{|b|}$ (see, e.g., [11]). Here, $b$ is the asymmetry parameter, defined as $b=-\cos (\Theta \pm$ $\eta) / \cos (\Theta \mp \eta)$. By choosing a proper asymmetry parameter $b$, one can tailor (in a limited range) the widths of Bragg reflections and the angular divergence of the reflected $\mathrm{x}$ rays to better suit certain applications. The increase in angular acceptance by this method is about a factor of 3 in the examples considered in this paper. This technique is widely used in x-ray optics. The optical scheme of the four-bounce cavity with asymmetrically cut crystals, shown in Fig. 6 is equivalent to the asymmetric $(+--+)$ monochromator introduced in [15].

We note here that asymmetric reflection, in addition to increasing the angular acceptance, is practically necessary, because diamond crystals considered in the following examples are usually manufactured in a few standard orientations.

\section{CRYSTAL CHOICE FOR SPECIFIC APPLICATIONS}

Crystals for the x-ray cavity for an XFELO, especially in four-crystal configuration, must have high reflectivity $R \gtrsim$ 0.95 . Such a high reflectivity at near-normal incidence can be obtained by using crystals composed of low- $Z$ atoms with high Debye temperature, such as $\mathrm{C}$ (diamond), $\mathrm{BeO}$, $\mathrm{SiC}$, or $\alpha-\mathrm{Al}_{2} \mathrm{O}_{3}$ (sapphire), etc. [11]. Both mentioned factors diminish the photoabsorption, which is the only cause of not a $100 \%$ reflectivity in perfect crystals. In particular, high Debye temperature ensures high DebyeWaller factors and thus small extinction, i.e., scattering length. Low $Z$ elements are favorable because of lower photoabsorption cross section. Among the commercially available crystals, diamonds are most promising in terms of achieving the highest reflectivity (Fig. 5) and in terms of 
TABLE I. Parameters of the diamond crystals in the four-bounce x-ray cavity (Fig. 6) optimized for the following applications: (a) 0.1 - to $1-\mathrm{meV}$ IXS with angular dispersive analyzers [11,27,28]; (b) $1 \mathrm{meV}$ IXS with segmented spherical Si(11 119) analyzers [26]; (c) 0.1 - to $1-\mathrm{meV}{ }^{57} \mathrm{Fe}$ NRS [21,22]. The thickness $d_{A}$ of crystal A is chosen to assure $4 \%$ out-coupling of x rays from the cavity.

\begin{tabular}{lccccccccc}
\hline \hline $\boldsymbol{H}(h k l)$ & $E_{H}[\mathrm{keV}]$ & $E_{0}[\mathrm{keV}]$ & $\Theta_{0}[\mathrm{deg}]$ & $\Delta \Theta[\mu \mathrm{rad}]$ & $\Delta E[\mathrm{meV}]$ & $d_{A}[\mu \mathrm{m}]$ & $\boldsymbol{n}(h k l)$ & $\eta[\mathrm{deg}]$ & Fig. 7 label \\
\hline (333) & 9.030 & 9.131 & 8.54 & 21 & 29 & 15 & $(001)$ & 63 & (a) \\
$(3311)$ & 20.489 & 20.514 & 2.83 & 5.5 & 5.5 & 75 & $(011)$ & 73 & (b) \\
$(337)$ & 14.4125 & 14.413 & 9.25 & 9 & 23 & 30 & $(011)$ & 68 & (c) \\
\hline \hline
\end{tabular}

ability to sustain high heat loads [16]. Crystal perfection is an issue, but with XFELO beams having a cross section of $\simeq 0.2 \mathrm{~mm} \times 0.2 \mathrm{~mm}$, small perfect crystals $\simeq 1-2 \mathrm{~mm}^{2}$ should be sufficient. Such crystals are available now $[17,18]$; however, the reflectivity of real crystals has yet to be studied.

XFELO will generate $\mathrm{x}$ rays in a meV bandwidth, and therefore would be an ideal source for high-energyresolution spectroscopies, such as inelastic x-ray scattering (IXS) $[19,20]$, nuclear resonant scattering (NRS) spectroscopies $[21,22]$, and bulk-sensitive hard X-ray photoemission spectroscopy (HAXPES) [23]. Imaging with hard $\mathrm{x}$ rays at near-atomic resolution $(\simeq 1 \mathrm{~nm})$ would also benefit from the high monochromaticity and low emittance of the XFELO beams [24], since multilayer Laue lenses, a most promising path towards nanofocusing [25], require hard $\mathrm{x}$ rays with a monochromaticity of $\Delta E / E \simeq 10^{-5}$. In the following we will consider particular designs of the four-bounced cavity optimized for these applications.

IXS spectrometers operate with $\mathrm{x}$ rays having photon energies close to the fixed energies $E_{0}$ of the IXS analyzers and require energy tunability of typically $\delta E_{0} \simeq 0.1 \mathrm{eV}$ (in rare cases several times more). In the most common version, segmented spherical analyzers are used, operating in almost exact backscattering geometry at the Bragg energies of $\mathrm{Si}(n n n)$ reflections [26]. It becomes problematic to find a good match between the diamond Bragg reflections

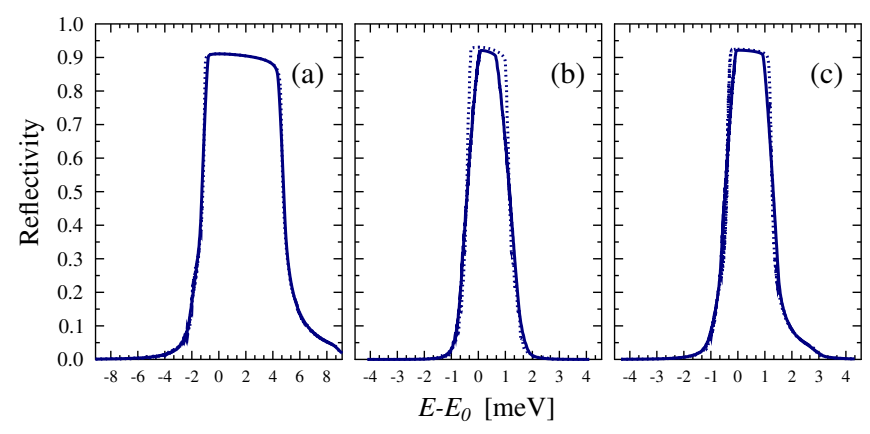

FIG. 7. (Color) X-ray photon energy dependence of the roundtrip reflectivity in the four-crystal XFELO cavity (Fig. 6). Crystal parameters are given in Table I, with Fig. 7 labels indicating the respective rows in the table. Calculations are performed with dynamical theory of x-ray diffraction as described in [11]. Incident beam divergence is $\Delta \Theta_{\mathrm{X}}=2.5 \mu \mathrm{rad}$ (solid lines) and $0.5 \mu \mathrm{rad}$ (dotted lines). and those of $\mathrm{Si}(n n n)$, with the condition given by Eq. (10) fulfilled. Instead, $\mathrm{Si}(111119)$ could be used as an analyzer with $1-\mathrm{meV}$ bandwidth at $E_{0}=20.5 \mathrm{keV}$, and with an advantage that this reflection matches well to the reflection C(3 311 ) for the diamond crystals in the four-crystal cavity. Table I shows the diamond crystal and the C(3 311$)$ Bragg reflection parameters optimized for the IXS spectrometer. Note that asymmetric reflections are used to better fulfill the condition imposed by Eq. (10). Figure 7(b) shows the energy dependence of the round-trip reflectivity in such a cavity. The peak reflectivity is $R \simeq$ 0.92 , and the energy bandwidth is $\simeq 1.5 \mathrm{meV}$ (FWHM).

Alternative angular dispersive IXS analyzers $[11,27,28]$, with $\mathrm{Si}(000)$ and dispersing element operating in the vicinity of $E_{0}=9.131 \mathrm{keV}$, have the potential of achieving $0.1-\mathrm{meV}$ resolution. Table I shows parameters of the crystals optimized for such a spectrometer. Figure 7(a) shows the energy dependence of the round-trip reflectivity in such a cavity. The peak reflectivity is $R \simeq 0.91$, and the energy bandwidth is $\simeq 6 \mathrm{meV}$ (FWHM).

Figure 7(c) shows the energy dependence of the roundtrip reflectivity for a four-bounce cavity operating in the vicinity of the energy $E_{0}=14.4125 \mathrm{keV}$ of the nuclear transition in ${ }^{57} \mathrm{Fe}$. The peak reflectivity is $R \simeq 0.92$, and the energy bandwidth is $\simeq 1.8 \mathrm{meV}$ (FWHM).

In the above, the diamond crystal platelets are assumed to be manufactured in one of the three standard orientations: (001), (011), and (111), as is usually the case. As the calculations presented in Fig. 7 show, due to the choice of the asymmetric reflections for the cavity crystals, the angular spread of $\mathrm{x}$ rays $\Delta \Theta_{\mathrm{X}}$ less than $2.5 \mu \mathrm{rad}$ does not affect the spectral properties of the cavity and its peak reflectivity.

\section{CONCLUDING REMARKS}

The tunability by the four-crystal scheme discussed here comes at the cost of greater complexity since it requires precise and coordinated translations and angular adjustments of four crystals. The requirement on angular stability of the crystals is especially tight, being about $\approx 10 \mathrm{nrad}$. A null-detection feedback system employed at the Laser Interferometer Gravitational Wave Observatory (LIGO) appears to be promising in achieving such a stability. The scheme, in which the correction signal is obtained by lockin amplification of the response of an oscillating input 
signal [29], has been recently implemented at the Advanced Photon Source beam line XOR/IXS 30-ID [30], providing an angular stability of $\simeq 50 \mathrm{nrad}$ for the monochromator's crystals. An additional factor of $5 \mathrm{im}-$ provement necessary for the XFELO cavity should be feasible.

Besides determining the mode size and angle in the interaction region, the choice of location and strength of the focusing elements also influences the mode stability and hence tolerances in the angular and positional stability of the crystals. The procedure for designing the $\mathrm{x}$-ray cavity parameters is similar to the well-known technique in accelerator physics [31], once it is recognized that the Rayleigh length plays the same role as the Courant-Snyder envelope function [32]. We then find that the use of two focusing elements in the tunable scheme is more versatile in enhancing the stability since there are more parameters to work with than the scheme using a single focusing mirror shown in Fig. 1 [14]. In fact, the latter scheme tends to be unstable because the distance between the mirror and the interaction point (about $100 \mathrm{~m}$ ) is far longer than the Rayleigh length (about $10 \mathrm{~m}$ ).

The alignment and tolerance issues will nevertheless be challenging for the four-crystal scheme discussed here. Assuming these technological challenges can be overcome, the scheme allows a significant enhancement in the capabilities of an XFELO.

\section{ACKNOWLEDGMENTS}

This work was supported by the U.S. Department of Energy, Office of Science, Office of Basic Energy Sciences, under Contract No. DE-AC02-06CH11357.

[1] R. Colella and A. Luccio, Opt. Commun. 50, 41 (1984).

[2] K. Kim, Y. Shvyd'ko, and S. Reiche, Phys. Rev. Lett. 100, 244802 (2008).

[3] D. Bilderback, C. Sinclair, and S. Gruner, Synchrotron Radiat. News 19, 30 (2006).

[4] P. Ostroumov, P. Piot, and K. Kim, in Proceedings of LINAC 08, 2008, to be published at http:// www.JACoW.org.

[5] Conceptual Design Report for the Linac Coherent Light Source, SLAC Technical Report No. SLAC-R-593, 2002.

[6] R. Brinkmann, in Proceedings of FEL06, 2006, p. 24, http://www.jacow.org.

[7] T. Tanaka, in Proceedings of FEL05, 2005, p. 75, http:// www.jacow.org.
[8] R. M. J. Cotterill, Appl. Phys. Lett. 12, 403 (1968).

[9] R. M. J. Cotterill, U.S. Patent No. 3518427, 1970.

[10] K. Kim, Argonne National laboratory Technical Report No. AAIA-PUB-2008-004, 2008.

[11] Y. Shvyd'ko, X-Ray Optics-High-Energy-Resolution Applications, Optical Sciences (Springer, Berlin, 2004), Vol. 98.

[12] A. Snigirev, V. Kohn, I. Snigireva, and B. Lengeler, Nature (London) 384, 49 (1996).

[13] B. Lengeler, C. Schroer, J. Tümmler, B. Benner, M. Richwin, A. Snigirev, I. Snigireva, and M. Drakopoulos, J. Synchrotron Radiat. 6, 1153 (1999).

[14] K. Kim, Argonne National laboratory, Technical Report No. AAIA-PUB-2008-004, 2008.

[15] M. Yabashi, K. Tamasaku, S. Kikuta, and T. Ishikawa, Rev. Sci. Instrum. 72, 4080 (2001).

[16] J.P.F. Sellschop et al., New Diamond Front. Carbon Technol. 10, 253 (2000).

[17] H. Sumiya, N. Toda, and S. Satoh, New Diamond Front. Carbon Technol. 10, 233 (2000).

[18] Y. Zhong, A. T. Macrander, S. Krasnicki, Y. S. Chu, J. Maj, L. Assoufid, and J. Qian, J. Phys. D 40, 5301 (2007).

[19] E. Burkel, Rep. Prog. Phys. 63, 171 (2000).

[20] M. Krisch and F. Sette, Light Scattering in Solids IX, Topics in Applied Physics (Springer, Berlin, 2007), Vol. 108, pp. 317-370.

[21] Special issue on Nuclear Resonant Scattering of Synchrotron Radiation, edited by E. Gerdau and H. de Waard [Hyperfine Interact. 123-125, 1999].

[22] R. Röhlsberger, Nuclear Condensed Matter Physics with Synchrotron Radiation (Springer, Berlin, 2004).

[23] See papers in Nucl. Instrum. Methods Phys. Res., Sect. A 547, 1 (2005).

[24] I. McNulty (private communication).

[25] H. Kang, J. Maser, G. Stephenson, C. Liu, R. Conley, A. Macrander, and S. Vogt, Phys. Rev. Lett. 96, 127401 (2006).

[26] C. Masciovecchio, G. Monaco, G. Ruocco, F. Sette, A. Cunsolo, M. Krisch, A. Mermet, M. Soltwisch, and R. Verbeni, Nucl. Instrum. Methods Phys. Res., Sect. B 111, 181 (1996).

[27] Y. V. Shvyd'ko, M. Lerche, U. Kuetgens, H. D. Rüter, A. Alatas, and J. Zhao, Phys. Rev. Lett. 97, 235502 (2006).

[28] Y. V. Shvyd'ko, U. Kuetgens, H. D. Rüter, M. Lerche, A. Alatas, and J. Zhao, AIP Conf. Proc. 879, 737 (2007).

[29] S. Witcomb (private communication).

[30] S. Stoupin, F. Lenkszus, R. Laird, K.-J. Kim, and Y. V. Shvyd'ko (to be published).

[31] E. D. Courant and H.S. Snyder, Ann. Phys. (Paris) 3, 1 (1958).

[32] Proceedings of the U.S. Particle Accelerator School [K.-J. Kim, AIP Conf. Proc. 184, 565 (1989)]. 